$\sin 8-71$

October 13, 1998

The Power and Limitations of Proportional Cutbacks in Common-Pool Resources

Roy Gardner

Departments of Economics and West European Studies, Indiana University

Workshop in Political Theory and Policy Analysis

Andrew Herr

Department of Economics, St. Vincent College

Workshop in Political Theory and Policy Analysis

\title{
Elinor Ostrom
}

Workshop in Political Theory and Policy Analysis, Indiana University

Center for the Study of Institutions, Population, and Environmental Change

James A. Walker

Department of Economics, Indiana University

Workshop in Political Theory and Policy Analysis

Presented at the Japan Science and Technology Conference "Game Theory and International Cooperation," Kyoto, Japan, March 24-27, 1998. We thank Akira Okada, Reinhard Selten, H. P. Young, Christopher Waller, the editor, and two anonymous referees for helpful comments. Financial support from National Science Foundation Grants \#SBR-9319835 and \#SBR-9521918 is gratefully acknowledged. 


\section{THE POWER AND LIMITATIONS OF PROPORTIONAL CUTBACKS IN COMMON-POOL RESOURCES}

\section{Abstract}

This paper examines the success and limitations of proportional cutbacks as an allocation rule for improving the performance of common pool resources (CPRs). Two field cases, one success and one failure, motivate the analysis. For symmetric CPRs, we establish the existence of efficiency-enhancing proportional cutbacks. We then introduce complications that arise in the presence of asymmetries, where there are high value types and low value types. This asymmetry induces a continuum of proportional cutbacks that raise efficiency above Nash equilibrium. Calibrating a linear-quadratic CPR model to global carbon dioxide emissions, the efficiency and distributional consequences of proportional cutbacks like those embodied in the Kyoto Protocol are derived. 
THE POWER AND LIMITATIONS OF PROPORTIONAL CUTBACKS IN COMMON-POOL RESOURCES

\section{INTRODUCTION}

Common-pool resources (CPRs) are natural or man-made resources in which: (a) exclusion is nontrivial due to significant physical or institutional barriers and (b) yield is subtractable (Ostrom, Gardner, and Walker, 1994). The general presumption is that individuals appropriating from CPRs are trapped in social dilemmas, leading inevitably to overuse and destruction of those resources (Gordon, 1954; Hardin, 1968). In recent years, scholars have documented a large number of field settings in which individuals face complex appropriation issues arising in CPR settings. Although many of these studies are consistent with the pessimistic presumption of overuse, there are a growing number of studies that document the capabilities of resource users to create endogenously and maintain institutions to facilitate more efficient use of the resources they use in common. ${ }^{1}$ The results from laboratory experiments, with the structure of finitely repeated CPR dilemmas, support the findings from the field. When subjects are placed in settings in which decisions are made in isolation, with minimal institutional structure, their aggregate behavior is generally consistent with equilibrium predictions of inefficient resource use. On the other hand, when allowed to communicate or use other coordinating mechanisms, subjects often adopt and maintain agreements consistent with efficient and sustainable resource use (Ostrom, Gardner, and Walker, 1994). Even in cases where the assets of participants are asymmetric, face-to-face communication allows participants to approach optimality (Hackett, Schlager, and Walker, 1994).

In many cases, resource users recognize their overuse only after the total of all of their withdrawals exceeds a sustainable level. Thus, some form of cutback is needed. In situations in which groups of resource users have coordinated their appropriation activities, a very simple institution has frequently been implemented — proportional cutbacks. Under such an institution, all resource users 
agree to reduce their appropriation activities by the same proportion. In cases where users are homogeneous in current usage, benefits received, and costs incurred, such an institution has strong appeal because there exists a proportional cutback that leads to an optimal outcome. However, in cases in which users are heterogenous with respect to important attributes related to use (current appropriation levels, benefits, costs, etc.), such an institution can lead to significant distributional conflict, and in some circumstances lead to a decrease in efficiency in resource use. When a few users obtain major benefits from restrictions on the appropriation levels of all users, the few face strong incentives to organize agreements to achieve long-term sustainable use of a resource. As such, they may be willing to bear most of the transaction costs of achieving an agreement to cut back and/or even assume a large proportion of the costs of a cutback themselves. Needless to say, when the users are sovereign nation states, the complications involved in reaching and sustaining any agreement are even greater than when the users are all members of a single sovereign state. Such situations are inherently asymmetric.

The impact of various kinds of heterogeneities on the likelihood of achieving more efficient results is now becoming a rich topic for analysis, both because of major policy concerns related to international agreements, but also because of recent theoretical work. In a pair of important articles, Baland and Platteau demonstrate that wealth inequality may promote or discourage efficient use of CPRs. In unregulated settings, the technology used for extraction is a key factor. In settings with convex production technologies (as we model below), wealth inequality is likely to exacerbate inefficiencies. When there are non-convexities (implying invidivisibility or threshold phenomena), wealth inequality can promote efficient use of the CPR (Baland and Platteau, 1997). Baland and Platteau (1998) also demonstrate that the efficiency gains from regulation will be lower as inequality among users increases. Dayton-Johnson and Bardhan (1998) examine the impact of unequal distribution of assets on the efficiency of outcomes. They find that increasing asset inequality is not associated with increased efficiency. On the other hand, once substantial inequality is the case, still 
further increases in the assets of one player may push participants closer to optimality. A curvilinear relation between asset inequality and economic efficiency is posited.

To better understand how a proportional cutback institution can arise and be maintained in the field, Section II provides a summary of two field cases, one (the Montreal Protocol) in which such an institution was successfully implemented and monitored, and another (EU fishing cutbacks) in which the success of the institution is yet to be proved. Moving from the case studies, section III examines the theoretical basis for proportional cutbacks to improve CPR performance. In particular, we establish the existence of a range of proportional cutbacks to move users from an inefficient game equilibrium to an improved outcome-in some cases, even an optimum. The basic criterion is that the proportional cutback be not too large, for which we give an explicit bound. Section IV develops a model for a parametric class of CPR situations to illustrate these results. We apply a specific calibration of the model to study the workings of proportional cutbacks in the Kyoto Protocol. Section V extends our analysis of one-shot games to time-dependent CPR situations, where users are heterogenous in benefits and prior appropriation rights to the CPR are present. The final section offers concluding comments.

\section{PROPORTIONAL CUTBACKS IN THE HELD}

In the late 1970's, satellite photos revealed for the first time the presence of ozone holes in the earth's atmosphere over the north and south poles. Atmospheric scientists immediately began researching probable causes for the ozone holes, as well as their likely implications for life on the planet. Since atmospheric ozone shields life on the planet from lethal radiation from space, the complete disappearance of ozone from the atmosphere would have catastrophic consequences for the biosphere.

Even worse, the ozone holes were observed to be expanding-with the ozone hole over the south pole covering almost the entire continent of Antarctica during the southern hemisphere's winter, 
and the hole over the north pole somewhat smaller. By 1985, atmospheric chemists had provided a causal explanation for the ozone holes beyond any reasonable doubt: chlorofluorocarbons (CFCs) used as propellants in aerosol cans and as coolants in air conditioners and refrigerators were the culprits. Each molecule of CFC, released at the earth's surface, had the capacity to rise to the ozone layer and chemically unbind over 4,000 molecules of ozone. CFCs, which had come to commercial prominence in this century, had in a short time created the ozone holes.

In response to the ozone situation, negotiations began on an international treaty to ban the commercial production of CFCs by all countries. In essence, this ban represents the limiting case of a proportional cutback - a 100\% cutback. Such cutbacks would certainly increase costs of refrigeration and air conditioning, both for producers and consumers. Moreover, new propellants would have to be developed for the multi-billion dollar aerosol can industry worldwide. The costs of these cutbacks would be concentrated in the developed countries, but they would also be felt in the developing countries. Treaty negotiations moved swiftly, culminating in the Montreal Protocol on Substances that Deplete the Ozone Layer, adopted by over 125 countries in 1987. The Montreal Protocol phases out the production and distribution of CFCs over a 10-year period-freon is just now disappearing from refrigerators and air conditioners in the United States (UNEP, 1991).

In the case of the ozone holes, the use of proportional cutbacks must be counted a considerable success. The holes themselves have stopped growing, although it is estimated it will take at least a century before they disappear altogether. The benefits to the quality of life on earth outweigh the losses to CFC producers and consumers. Indeed, the latter have succeeded in developing close substitutes for CFCs without serious atmospheric consequences.

Not all international attempts to use proportional cutbacks are as successful as the Montreal Protocol. To cite just one example, consider the common fisheries policy of the European Union (EU). Persistent overfishing of EU waters has led to serious declines in the population of species such as cod, herring, mackerel, sole, sardines, and salmon. Fishing at current levels is clearly 
unsustainable, and could lead to commercial extinction of all the above species in EU waters. Indeed, the problem has been so severe as to lead to international incidents involving EU member countries and other countries (UK vs. Iceland, France vs. Canada, Spain vs. Canada), as well as tensions between EU members.

In an attempt to address the situation, the European Commission in 1996 proposed a proportional cutback of $40 \%$ in the fishing fleets of all EU member countries, to be phased in over 6 years. These cutbacks were attacked by various countries, and the Commission withdrew the plan rather than see it vetoed. The discussion exhibited a sharp difference between rich and poor fishing countries-with Spain and Portugal wanting little if any reduction in their fleets, while the UK wanted even larger reductions in Spanish and Portuguese fleets.

In April of 1997, the European Commission brought forward a revised proportional cutbacks plan. Under this plan, fishing fleets are to be differentially reduced, depending on the severity of the overfishing. For species in the category "most endangered," fishing fleets are to be cutback by $30 \%$ over the next five years. This category includes Atlantic salmon and Atlantic swordfish. For species in the category "overfished," fleets are to be cutback by $20 \%$ over that same period. For all other species, the size of the fishing fleet is frozen. The European Council decided this revised plan by weighted majority rule. France and the UK, both of whom still in the opposition, were outvoted by the rest of the member states (Pesca, 1998)

It is still too early to tell if the EU's adoption of a variety of proportional cutbacks, after 40 years of overfishing, will lead to a comeback in commercial fish populations. In this regard, the experience of Canada, which used similar cutbacks in an attempt to save its "most endangered" cod fishery, is hardly cause for optimism. The Canadian cod fishery, despite 25 years of fisheries policy attempting to sustain it, is now commercially extinct [Finlayson and McCay, 1998].

The difficulties facing the EU are even greater, given the international dimension. Consider the most endangered Atlantic swordfish. The current international agreement calls for only 11,300 
tons to be taken in a given year--a rough estimate of sustainable use. By contrast, the actual use is 16,000 tons/year--half of which is taken by EU members. Proportional cutbacks on the part of all countries involved in this fishery (USA, Canada, Japan, EFTA members) of roughly $30 \%$ are called for to restore the agreed level. Even if the EU were to cut its use back by $30 \%$, from 8,000 tons to 5,600 tons, that would still only go half way to solving the Atlantic swordfish problem.

These cases illustrate situations in which resource users have adopted proportional cutback institutions in an attempt to reduce overall appropriation levels. The theoretical results of the next two sections examine the extent to which such a simple institution as proportional cutbacks can lead to desirable efficiency and distributional consequences.

\section{CPR GAME MODEL}

The next two sections consider one-shot CPR games, where the impact of proportionalcutbacks can be most readily understood. For an analysis of corresponding time-dependent supergame results, see [Herr, Gardner, and Walker, 1997].

Consider a CPR normal form game with $n$ players. Each player $i$ has a strategy $x_{i}$ drawn from the interval $\left[0, x_{\max }\right]$. Let

$$
x=\left(x_{1}, \ldots, x_{n}\right)
$$

denote a vector of strategies, one for each player. Let $\mathbf{x}_{\mathbf{i}}$ denote the vector of strategies not including $x_{i}$. Each player has a payoff function $u_{i}(x)$ which depends on the entire vector of strategies $x$. Assume the following:

$u_{i}(x)$ is concave for all $i$.

We further assume, simply for purposes of exposition, that

$u_{i}(x)$ is twice differentiable.

The following is an opt-out assumption:

$$
u_{i}\left(x_{i}=0, x_{i}\right)=0 \text { for all } x_{j} .
$$

A player who does not access the commons receives a zero payoff. 
The following is a convenient normalization:

$$
u_{i}(0)=0 .
$$

If all players opt out of the commons, the payoff is zero to each.

The following negative externality assumption is substantive:

$$
\partial \mathbf{u}_{\mathrm{i}} / \partial \mathbf{x}_{\mathrm{j}}<0
$$

An increase in use by another player reduces a given player's payoff.

Let $\mathbf{x}^{\circ}$ denote an optimal solution to the game. More specifically, $\mathbf{x}^{\circ}$ maximizes

$\Sigma u_{i}(x)$ over all vectors $\mathbf{x}$ in $\left[0, x_{\max }\right]^{n}$. By compactness of the strategy set and the continuity of concave functions, such an optimum exists; moreover, if there are multiple optima, one of them is symmetric. In this discussion, we restrict attention to the symmetric optimum. If the optimum is interior $\left(x_{\max }>x^{*}>0\right)$, the usual necessary and sufficient conditions apply.

Let $\mathbf{x}^{e}$ denote an equilibrium to the game. For player $i, x_{i}^{e}$ maximizes $u_{i}\left(x_{i}, x_{j}{ }^{\circ}\right)$ over all $x_{i}$ in $\left[0, x_{\max }\right]$. A routine fixed point argument establishes that an equilibrium exists. Since the game is symmetric, at least one equilibrium is symmetric. ${ }^{2}$

Throughout, restrict attention to an interior equilibrium $x^{*}\left(x_{\max }>x^{e}>0\right)$ and an interior optimum. We first show that a Nash equilibrium is not an optimum. To see this, consider the first order characterization of an optimum:

$$
0=\Sigma \partial u_{j} / \partial x_{i}=0 \quad \text { for all } i \text {, for every } j
$$

At a Nash equilibrium, the term in this sum

$$
\partial u_{j} / \partial x_{j}=0
$$

Since the remaining terms in the sum are negative from the negative externality condition, an equilibrium cannot be an optimum.

Moreover, we can establish the following overuse condition: ${ }^{3}$

$$
\mathbf{x}^{*}<\mathbf{x}^{*}
$$

We will actually show more, namely that proportional cutbacks, as long as they are small, will lead to 
an improvement in efficiency.

Consider a small reduction in strategies by all players:

$$
\mathrm{dx}_{\mathrm{i}}<0 \quad \text { for all } \mathrm{i}
$$

this reduction taking place at a Nash equilibrium. Taking the total differential of utility for an arbitrary player $\mathrm{i}$,

$$
d u_{i}=\partial u_{i} / \partial x_{i}+\Sigma \partial u_{i} / \partial x_{j}>0
$$

again using negative externality and Nash equilibrium.

Since this is true in general for $d x<0$, it is certainly true for

$$
d x_{i} / x_{i}=d x_{j} / x_{j}=1-s
$$

Here 1-s is a proportional cutback, and $s$ is a scalar such that

$$
0 \leq s \leq 1
$$

What the scalar $s$ does is contract a Nash equilibrium towards the origin:

$\lim s x^{e}=0$

as $s$ tends towards 0 .

To see how proportional cutbacks work, consider the measure of efficiency efficiency $=\Sigma u_{i}\left(s x^{c}\right) / \Sigma u_{i}\left(\mathbf{x}^{0}\right)$.

The denominator of this function is the optimal value of the CPR. The numerator is a function of the scalar $s$ alone. When $s=1$, we get the efficiency of Nash equilibrium. When $s<1$, we get the efficiency of a proportional cutback from Nash equilibrium.

Since only the numerator is variable, we focus attention on it:

$$
f(s)=\Sigma u_{i}\left(s x^{e}\right)
$$

The function $f(s)$ is concave in $s$ (this follows from concavity of the payoff function and is easily seen by taking two derivatives). Further, $\mathrm{f}(\mathrm{s})$ is increasing to the left in the vicinity of $s=1$, as we just showed. These two facts lead to the following observation: $f(s)$ is a concave function which is increasing immediately to the left of $s=1$, and which attains the value $f(0)=0$ at $s=0$ (this 
follows from normalization). ${ }^{4}$

Since $f(s)$ is concave, it is continuous. By the intermediate value theorem, there exists a critical value $s^{\mathfrak{c}}$ such that

$$
f\left(s x^{e}\right)=f\left(1 x^{*}\right)
$$

At values of $s$ between

$$
s^{c} \leq s \leq 1
$$

we have

$$
\left(f s x^{*}\right) \geq f\left(1 x^{*}\right)
$$

so that proportional cutbacks in this critical region improve efficiency. Proportional cutbacks, as long as they are not too large, improve efficiency, as we promised to show.

Now consider the implications for efficiency. Since $f(s)$ is continuous, it must achieve its maximum value on the compact set $\left[s^{c}, 1\right]$. Denote this maximum value $f\left(s^{*}\right)$, and let $s^{*}$ be a contraction factor $\left(\left(1-s^{*}\right)\right.$ being the corresponding proportional cutback) that achieves the maximum value $\mathrm{f}\left(\mathrm{s}^{*}\right)$.

There are two cases to consider, the first best and the second best. Suppose that both the Nash equilibrium and the optimum are symmetric, as would be the case if this were a symmetric game with a unique equilibrium. In the first best case, the scalar $s^{*}$ achieves the optimal value:

$$
f\left(s^{*} x^{e}\right)=f\left(x^{0}\right)
$$

since the maximum sum of utilities is attained at $s^{*} x^{*}$ as well as at the optimum. We call such a proportional cutback optimal, since it leads to an optimal solution.

In the second best case, which we would expect to hold in an asymmetric game, an optimal proportional cutback is no longer available. Still, any small proportional cutback-where small means in the range between 0 and $1-\mathrm{s}^{\circ}$, will improve efficiency relative to Nash equilibrium:

$$
\Sigma u_{i}\left(s x^{e}\right) \geq \Sigma u_{i}\left(x^{*}\right)
$$

The best of all such proportional cutbacks--the second-best proportional cutback--is again given by 1- 
$s^{*}$. In this case, the efficiency achieved by the proportional cutback $1-s^{*}$ is less than $100 \%$. How much less than $100 \%$ is the efficiency achieved depends on the specific CPR being used.

The above efficiency analysis has been carried out completely in terms of the entire CPR. However, the same analysis can be carried out at the level of the individual user, and all the above results go through as before.

Now define the function

$$
f_{i}(s)=u_{i}\left(s x^{*}\right)
$$

This function represents player i's efficiency function--player i's preferences over proportional cutbacks, since the optimal value of the resource is constant. In particular we can define the scalar $\mathrm{s}_{\mathrm{i}}{ }^{*}$ and proportional cutback $1-s_{i}^{*}$ which player $i$ finds best for i's own payoff. This is the scalar such that

$$
f_{i}\left(s_{i}^{*}\right)=\max f_{i}(s)
$$

For each player $i$, there is a (second)best proportional cutback $1-\mathrm{s}_{i}^{*}$. We cannot rule out a priori that some player i may find a proportional cutback best which is larger than $1-s^{c}$. Indeed, we construct examples of such preferences in the next section.

Notice that the issue space of proportional cutbacks is the 1 -dimensional interval $[0,1]$. It follows from concavity of the functions $f_{i}(s)$ on this interval that preferences of the entire set of players are single-peaked. Thus, we have all the elements necessary for the Median Voter Theorem. In the event that players vote over proportional cutbacks using majority rule, then the voting equilibrium of that election is the proportional cutback favored by the median voter.

\section{CPR Game with Two Player Types}

Now extend the above model to allow explicitiy for a single asymmetry between players. Consider the case in which there are two player types, a high value type (H-type) and a low value type (L-type). For ease of exposition, we will assume that there is one player of each type, $n_{H}=n_{L}$ $=1$. This is again relaxed in the next section. The strategy vector $\mathbf{x}$ is given by 


$$
x=\left(x_{H}, x_{L}\right)
$$

Both player types continue to have the same strategy set as before, but with different payoff functions $u_{L}(x)$ and $u_{H}(x)$. Assume that the $H$-type is favored in the sense that for any strictly positive strategy vector $x$ with $x_{H}=x_{L}$,

$$
u_{H}(x)=\left(a_{H}-a_{L}\right) x_{H}+u_{L}(x)>u_{L}(x)
$$

where $a_{H}-a_{L}>0$. Inequality (1) guarantees that a high type earns more than a low type at both an equilibrium and an optimum. We maintain all the other previous assumptions on both types of payoff functions.

Denote an optimal solution to this game by $x^{\circ}$. Existence of an optimal solution follows from compactness of the strategy space and concavity (hence continuity) of the payoff functions. In general, $\mathbf{x}^{\circ}$ will not be symmetric; indeed, for all interior optima, one has from (1) that

$$
x_{H^{0}}>x_{L}^{0} \text {. }
$$

Similarly, denote by $\mathbf{x}^{*}$ an equilibrium to the game. We again have

$$
x_{H}{ }^{*}>x_{L}{ }^{e}
$$

We continue to assume that (1) holds, so that proportional cutbacks have at least intuitive appeal. It is clear that the above analysis involving the critical value $s^{e}$ and the critical value of a proportional cutback $1-s^{c}$ applies to this asymmetric game. Proportional cutbacks which are not too large will enhance efficiency in a CPR.

\section{A PARAMETRIC CLASS OF CPRS}

The parametric class of CPRs considered in this section is based on a CPR game model used by Gardner, Moore, and Walker (1997) and Herr, Gardner, and Walker (1997). ${ }^{5}$ In the interpretation here, strategies take the form of emissions, and payoffs are either linear or quadratic in all strategies, and represent the amount of opportunity cost reduction, relative to having to store emissions on (or beneath) the earth's surface.

\section{Symmetric CPR Game: An Explicit Optimal Cutback}


Consider a CPR game played by n players. Each player $\mathrm{i}$ has the payoff function

$$
u_{i}(x)=a x_{i}-b x_{i}^{2}-x_{i}\left(c_{0}+k X / 2\right) \text {. }
$$

where group emissions are given by $X=\Sigma x_{i}$. Notice that (9) satisfies all the assumptions of the previous section: concavity, opt-out, and normalization. In the normal form game defined by (9), it is profitable for players to generate emissions into the CPR as long as a $>c_{0}$, which we maintain. A routine calculation shows that the game equilibrium is given by

$$
x_{i}^{0}=\left[a-c_{0}\right] /[2 b+(n+1) k / 2]
$$

while the optimum is given by

$$
x_{i}^{0}=\left[a-c_{0}\right] /[2 b+n k]
$$

We can solve explicitly for the optimal cutback, arriving at

$$
s^{\circ}=[2 b+(n+1) k / 2] /[2 b+n k]
$$

It is worth pointing out that $\mathrm{s}^{\circ}$ is monotonically decreasing with $\mathrm{n}$, and approaches the limit onehalf as n goes to infinity. In this symmetric CPR with a large number of players, the optimal cutback is roughly $50 \%$.

\section{Asymmetric CPR Game: A Continuum of Proportional Cutbacks}

Consider the game that results when a single asymmetry is introduced into this game via the parameter, a, of the benefit function. Again, assume that there are only two types of players, high value types (H-types) and low value types (L-types). The payoff function defining high value types includes the parameter $a_{H}$, while the benefit function defining low value types includes the parameter $a_{L}$, with

$$
a_{H}>a_{L} \text {. }
$$

Each player type faces the same cost function, given by (8). We have the payoff functions

$$
\begin{aligned}
& u_{H}(x)=a_{H} x_{H}-b x_{H}^{2}-x_{H}\left(c_{0}+k X / 2\right) \\
& u_{L}(x)=a_{L} x_{L}-b x_{L}^{2}-x_{L}\left(c_{0}+k X / 2\right)
\end{aligned}
$$

where $n_{H}$ denotes the number of high-type agents; $n_{L}$, the number of low-type agents; $X_{H}=n_{H} x_{H}$, the 
total emissions by high value types; $X_{L}=n_{L} X_{L}$, the total emissions by low value types; and $X=X_{H}+$ $X_{h}$, total emissions. It is clear that (12) satisfies condition (6).

We assume hereafter that all optima and equilibria are interior. ${ }^{6} \mathrm{~A}$ routine calculation shows the optimal to be given by:

$$
\begin{gathered}
x_{H^{0}}=\left[\left(a_{H^{-}}-c_{0}\right)+\left(a_{H}-a_{L}\right) k n_{L} /(2 b)\right] /[2 b+k n] \\
x_{L}^{0}=\left[\left(a_{L}-c_{0}\right)-\left(a_{H}-a_{L}\right) k n_{H} /(2 b)\right] /[2 b+k n]
\end{gathered}
$$

Given the assumption that $a_{H}>a_{L}$, (13) implies that high value types emit more into the CPR and get higher payoffs at the optimum than do low value types.

When $\mathbf{n}>1$, the optimal solution of the CPR game is not an equilibrium.

A routine calculation yields the interior game equilibrium:

$$
\begin{aligned}
& x_{H}{ }^{e}=\left[\left(a_{H}-c_{0}\right)+\left(a_{H}-a_{L}\right) k n_{L} /(4 b+k)\right] /[2 b+k(n+1) / 2] \\
& x_{L}{ }^{e}=\left[\left(a_{L}-c_{0}\right)-\left(a_{H}-a_{L}\right) k n_{H} /(4 b+k)\right] /[2 b+k(n+1) / 2]
\end{aligned}
$$

Again, given the assumption that $a_{H}>a_{\ell},(14)$ implies that high value types emit more into the CPR and earn higher payoffs at the equilibrium than do low value types.

Comparing the optimum (13) to the equilibrium (14), we easily verify inequality (1). The game equilibrium uses more of the CPR than is optimal. In addition, we can show that the ratio of high-value type to low value type usage at equilibrium is lower than that at optimum:

$$
x_{H}{ }^{0} / x_{L}{ }^{0}<x_{H}{ }^{0} / x_{L}{ }^{0} \text {. }
$$

(15) means that the optimal proportional cutback--the one that converts an equilibrium to an optimum according to (2), is not available in this parametric class of CPRs.

We now exhibit a continuum of proportional cutbacks satisfying (3). Explicitly solving (4) for the critical value $s^{c}$, we get

$$
s^{c}=(A / B)-1
$$

where

$$
A=n_{H} x_{H}^{\circ}\left(a_{H}-c_{0}\right)+n_{L} x_{L}^{*}\left(a_{L}-c_{0}\right)
$$




$$
B=n_{H} x_{H}{ }^{\circ}\left(b x_{H}+k X^{\circ} / 2\right)+n_{L} x_{L}^{e}\left(b x_{L}{ }^{e}+k X^{c} / 2\right)
$$

At an interior equilibrium, the critical value $s^{\circ}$ lies strictly between 0 and 1 , guaranteeing the existence of an interval of proportional cutbacks (5) that enhance efficiency. In particular, this interval includes the proportional cutback $\left(1-\mathrm{s}^{*}\right)$ that maximizes the sum of payoffs along the ray through the game equilibrium. The $\mathrm{s}^{*}$ corresponding to this proportional curback is given by

$$
s^{*}=(\mathrm{A} / 2 \mathrm{~B})
$$

where $A$ and $B$ are the same as given above. We have $s^{c} \leq s^{*}$, and the interval of proportional cutbacks

$$
1-s^{e}>1-s \geq 0
$$

which includes 1 - $\mathrm{s}^{*}$.

\section{An Illustration of Proportional Cutbacks}

In the following example, the proportional cutbacks and their efficiency and payoff implications are discussed for a model based on the carbon dioxide $\left(\mathrm{CO}_{2}\right)$ emissions problem, the subject of an international conference in Kyoto in December of 1997. In this interpretation, $X^{\circ}$ represents global carbon dioxide emissions at 2010 levels. The players are countries, or groups of countries. There are two types of players, developed countries (the high value type) and developing countries (the low value type). The ability to emit and store $\mathrm{CO}_{2}$ in the atmosphere is considerably more valuable to a developed country than to a developing country. We capture this with the parameter values

$$
\mathrm{a}_{\mathrm{H}}=1.00, \mathrm{a}_{\mathrm{L}}=0.70 .
$$

Thus, it is worth $30 \%$ more to a developed country to store its $\mathrm{CO}_{2}$ emissions in the atmosphere, given the opportunity cost of the alternative of storing these emissions on or below the surface, than the same emission and storage is worth to a developing country.

Identify 3 high value countries or groups of countries: USA, Europe, and Japan. Divide the low value countries into 5 emissions groups: PRC, former soviet union (FSU), rest of Asia, Africa, and Latin America. This implies 


$$
\mathbf{n}_{\mathrm{L}}=5, \mathrm{n}_{\mathrm{H}}=3
$$

Finally, set

$$
\mathrm{b}=\mathrm{k}=c_{0}=0.01
$$

Computing game equilibrium, the total emissions level is

$$
\mathrm{X}^{*}=98.8
$$

$65 \%$ of which is emitted by the high value countries--a percentage which calibrates fairly well with current global $\mathrm{CO}_{2}$ emissions. The efficiency of the game equilibrium is $74 \%$. By contrast, at the optimum, we get a total emissions level of

$$
X^{\circ}=64.2
$$

a reduction of some $35 \%$. This reduction is not shared equally, however--as follows from (15). The ratio of high-type to low-type usage is 2.5 at the game equilibrium, but 7.25 at the optimum--meaning that developing countries cut back more than do developed countries.

There is a broad consensus among atmospheric scientists that a sustained reduction in $\mathrm{CO}_{2}$ emissions by this amount sometime in the near future would maintain the current $\mathrm{CO}_{2}$ level in the atmosphere. By the same token, $\mathrm{CO}_{2}$ emissions at current levels projected into the future will double the $\mathrm{CO}_{2}$ atmospheric concentration sometime in the next century--with considerable impact on global climate change, sea levels, and patterns of agricultural production.

Now consider proportional cutbacks in the range from $0 \%$ to $64 \%$ (the latter being the critical value for this calibration), in light of the Kyoto Protocol. The average proportional cutback agreed to by Japan, the United States, and Europe was a 7\% reduction of $\mathrm{CO}_{2}$ emissions from 1990 levels, to be achieved by the year $2010 .^{7}$ At current trends, this amounts to roughly a $30 \%$ reduction from projected levels in the year 2010. Interestingly, the payoff-maximizing cutback $1-s^{*}$ in this calibration is $32 \%$, and it achieves a very high efficiency, $97 \%$. This gain in efficiency is especially impressive, given the simplicity of the institution achieving it. ${ }^{8}$

One of the sensitive features of the Kyoto Protocol is that its proposed proportional cutbacks 
only apply to the 33 developed countries (the so-called Index I countries) at the outset. Although there are provisions for including the developing countries in the cutbacks later, those countries do not participate at the present time. This feature — a glaring lack of proportionality-makes it unlikely that the U.S. Senate will approve the Protocol in the election year 1998. For this reason, the Clinton administration has postponed bringing the Protocol to the Senate for approval until 1999 at the earliest.

These results-in particular the high efficiencies achieved by proportional cutbacks in the range from $20 \%$ to $40 \%$-are robust to a wide variation in the underlying parameter values. This is a CPR situation in which proportional cutbacks have attractive second-best properties, which are only enhanced if a system of global trading permits is appended to them.

The biggest issues for signatories of the Kyoto Protocol are monitoring and enforcement of the agreement—an area in which international agreements historically have been less than perfect (Choucri, 1993; Haas et al, 1993).

V. Proportional Cutbacks in a Time-Dependant Model

So far we have demonstrated the range of values of proportional cutbacks that improve CPR performance in a one-shot game. The discussion of field cases, as well as of the Kyoto Protocol, has suggested the importance of the time element in the efficiency and sustainability of CPRs. This section suggests a way of extending the above results to a time-dependant CPR game. In this context, aggregate emissions at time $\mathrm{t}, \mathrm{X}(\mathrm{t})$, is given by

$$
X(t)=\Sigma x_{i}(t)
$$

where $x_{i}(t)$ denotes player $i$ 's emissions at time $t . X(t)$ is a function of time $t$, from $t=0$ to $t=T$, where the horizon $\mathrm{T}$ tends to infinity. In such a setting, a proportional cutback--a scalar--would seem unequal to the task of achieving anything close to an optimal solution. In this section, we show that proportional cutbacks as a function of time--just as they are used in the Montreal and Kyoto 
Protocols--can produce an optimal solution in a symmetric game, and improve performance in an asymmetric game.

To keep matters as simple as possible, suppose that all users of the CPR are symmetric, with an instantaneous benefit function at time $\mathrm{t}$ given by analogy from (9):9

$$
\begin{aligned}
& u_{i t}\left(x_{i}\right)=B_{i}\left(x_{i}(t)\right)-C_{i}\left(x_{i}(t), X(t)\right) \\
& =a x_{i t}-b x_{i t}^{2}-x_{i n}\left(c_{0}+k X(t) / 2\right) .
\end{aligned}
$$

Denote by $d(t)$ the state of the CPR at time t. The CPR evolves according to

$$
d(t+1)=d(t)+h-X(t)
$$

where $h$ is a constant renewal parameter.

Suppose that $\mathbf{x}^{\circ}(t)$ is the time path of the optimal solution, with each player having the individual optimal solution $x_{i}{ }^{\circ}(t)$. For a symmetric equilibrium time path given by $x_{i}{ }^{e}(t)$, define the scalar function of time $s^{\circ}(t)$ by

$$
s^{0}(t) x_{i}^{c}(t)=x_{i}^{o}(t)
$$

in analogy to (2). Then the optimal proportional cutbacks, as a function of time, are $1-s^{\circ}(t)$. In a symmetric world, optimal proportional cutbacks do exist.

As a simple example, suppose sustainability is optimal:

$$
X^{e}(t)=h
$$

following from (17). Then we have

$$
x_{i}^{\circ}=h / n
$$

This would apply to atmospheric balance of CFCs in the case of the Montreal Protocol and atmosphere balance of $\mathrm{CO}_{2}$ in the case of the Kyoto Protocol-if all countries were symmetric.

The presence of asymmetries complicates matters considerably. Given the second-best nature of proportional cutbacks, the optimal proportional cutback as a function of time is no longer available. Only second-best proportional cutbacks are available. Let $x^{e}(t)$ denote the equilibrium path. Typically, the equilibrium path will use the CPR more heavily than the optimal path early, reversing the pattern 
later (for instance, along an equilibrium leading to exhausting or extinction of the resource in finite time). Cutbacks in such a situation only make sense early along the path. Suppose that time $t^{f}$ is the critical time: for $t<t$, (1) holds:

\section{$X^{\circ}(t)>X^{\circ}(t)$}

In the time interval before $\mathrm{t}^{\mathrm{c}}$, a range of cutback functions will still work to improve efficiency. For instance, let $s^{*}(t)$ be the scalar function that maximizes the sum of payoffs along the path $\mathrm{sx}^{\mathrm{e}}(\mathrm{t})$, subject to the transversality condition that the state of the resource $d\left(t^{c}\right)$ be the same as at that optimal path at that time. Then the proportional cutbacks $1-\mathrm{s}^{*}(\mathrm{t})$ until the critical time $\mathrm{t}^{\mathrm{c}}$ will serve to enhance efficiency, just as their scalar analogue $1-s^{*}$ does in the one-shot game.

In one respect, the countries in the atmospheric cases have been fortunate- the large users have also been the high value users. Suppose instead that the large users were low value users, and that they had historically established rights to the CPR such as in the case of territorial fishing waters of EU members. In that event, the low-value users are able to impose proportional cutbacks restricted only to the high-value users. In the extreme case, cutting high-value users completely from the CPR must make efficiency suffer. With a large enough payoff asymmetry, an efficiency loss from such a differential type of proportional cutback is assured. One has only to compare the optimal value of a CPR exploited only by low-value users to the optimal value of the same CPR exploited only by highvalue users, to establish an upper bound on the efficiency loss. This kind of asymmetry may help account for the difficulty the EU has had in arriving at successful proportional cutbacks for its fisheries CPR.

\section{CONCLUSION}

This paper studies the success and limitations of proportional cutbacks for improving the performance of common pool resources (CPRs) which cross national boundaries. For a general class of CPRs, we demonstrate the power of proportional cutbacks for the case where users are symmetric in all relevant characteristics. In such cases, there exists an optimal proportional cutback-one which 
transforms a symmetric game equilibrium into an optimum. When an optimal proportional cutback is not available - as is often the case in asymmetric games-we exhibit the existence of a range of proportional cutbacks which enhance performance. In general, proportional cutbacks enhance performance as long as they are not too large.

The theoretical results developed in this paper have implications for interpreting case studies where proportional cutbacks have been implemented, and for policy analysis. A case such as the Montreal Protocol, discussed above, involves a multiplicity of asymmetries-not just two.

Nevertheless, it is remarkable that the agreement negotiated by the nations of the world and selfenforced has survived for over a decade. This is strong evidence for countries' own interests' being served by adhering to the agreement. In cases involving the failure of proportional cutbacks, either to be agreed or to be adhered to, one should be on the lookout for cutbacks that were too large to be beneficial, or asymmetries in payoffs where cutbacks damaged the long run interests of some of the countries involved.

One issue which needs further work is the process by which countries in asymmetric CPR settings devise and sustain over time rules for improving CPR performance. It is not clear, for example, that if one took a mechanism design approach, proportional cutbacks would be the institutional outcome. Such mechanisms do appeal to simplicity and fairness-representing a kind of compromise between high- and low-value users. Further documentation of case studies, such as the ongoing debate over the Kyoto Protocol, may help to clarify just how such rules are devised in the field, while controlled laboratory experiments can be explicitly designed to address such issues. 
1. National Research Council, 1986; McCay and Acheson, 1987; Fortmann and Bruce, 1988; Wade, 1988; Berkes, 1989; Pinkerton, 1989; E. Ostrom, 1990; Sengupta, 1991; Blomquist, 1992; Bromley et al., 1992; Tang, 1992; Thomson, 1992; Dasgupta and Mäler, 1992; V. Ostrom, Feeny, and Picht, 1993; Netting, 1993; and E. Ostrom, Gardner, and Walker, 1994. See also items cited in Martin, 1989/1992;).

2. The above argument goes through for the case of multiple symmetric equilibria, each of which will have its own proportional cutback satisfying (2).

3. For a discussion of appropriation problems, and a full array of examples, see Ostrom, Gardner, and Walker (1994).

4.This shows that the normalization assumption does have impact. Applied to the situation in the Montreal Protocol, it says that $\mathrm{f}(\mathrm{s})$ reaches its maximal value at $\mathrm{f}(0)$.

5. This specification is general enough to encompass most of the models in Ostrom, Gardner, and Walker (1994). See Herr (1996) for a detailed examination of the similarities of these models.

6. A detailed analysis of the corner equilibria and optima is available from the authors upon request.

7. The exact figure for the USA is $7 \%$; Europe at $6 \%$ and Japan at $8 \%$ are $1 \%$ away from the average.

8. Gains to an agreement of this magnitude are also reported by Escapa and Gutierrez (1997).

9. In this context, a payoff asymmetry as in $a_{H}>a_{L}$ has two effects. In any given time period, the high-value type extracts at least as much of the resource as the low-value type-just as we have seen in the one-shot game. In addition, a high-value type may extract longer than a low-value type, since a low-value type will hit the corner condition (20) sooner than a high-value type will. 
$\underline{\text { References }}$

Baland, Jean-Marie, and Jean-Philippe Platteau. 1997. "Wealth Inequality and Efficiency in the Commons. The Unregulated Case." Oxford Economic Papers. 49(4): 451-482.

Commons. The Regulated Case." Oxford Economic Papers. 50(1): 1-32.

1998. "Wealth Inequality and Efficiency in the

Berkes, Fikret, ed. 1989. Common Property Resources: Ecology and Community-Based Sustainable Development. London: Belhaven Press.

Bromley, Daniel W., David Feeny, Margaret McKean, Pauline Peters, Jere Gilles, Ronald Oakerson, C. Ford Runge, and James Thomson, eds. 1992. Making the Commons Work: Theory, Practice, and Policy. San Francisco, Calif.: ICS Press.

Cave, Jonathan, and Stephen W. Salant. 1995. "Cartel Quotas under Majority Rule," American Economic Review 85:82-102.

Choucri, Nazli. 1993. "Political Economy of the Global Environment." International Political Science Review. 14:103-116.

Dasgupta, Partha, and Karl Göran Mäler. 1992. The Economics of Transnational Commons. Oxford: Clarendon Press.

Dayton-Johnson, Jeff and Pranab Bardhan. 1998. "Inequality and Conservation on the Local Commons: A Theoretical Exercise." Berkeley: University of California, Department of Economics Working Paper.

Escapa, Marta and Maria Jose Gutierrez. 1997. "Distribution of Potential Gains from International Environmental Agreements: The Case of the Greenhouse Effect." Journal of Environmental Economics and Management. 33:1-16.

Eswaran, Mukesh and Tracy Lewis. 1984. "Appropriability and the Extraction of a Common Property Resource," Economica 51:393-400.

Finlayson, Christopher and Bonnie J. McCay. 1998. "Crossing the Threshold of Ecosystem Resilience: The Commercial Extinction of Northern Cod." In Firket Berkes and Carl Folke (eds) Linking Social and Ecological Systems: Management Practices and Social Mechanisms for Building Resilience. Cambridge: Cambridge University Press, 311-338.

Fortmann, Louise, and John W. Bruce, eds. 1988. Whose Trees? Proprietary Dimensions of Forestry. Boulder, Colo.: Westview Press.

Gardner, Roy, Michael Moore, and James A. Walker. 1997. "Governing a Groundwater Commons: A Strategic and Laboratory Analysis of Western Water Law," Economic Inquiry 35:218-234.

Gordon, H. Scott. 1954. "The Economic Theory of a Common Property Resource: The Fishery," Journal of Political Economy 62:124-42.

Haas, Peter M., Robert O. Keohane, and Marc A. Levy, eds. 1993. Institutions for the Earth: Sources of Effective Environmental Protection Cambridge, MA: MIT Press. 
Hackett, Steven, Edella Schlager and James Walker. 1994. "The Role of Communication in Resolving Commons Dilemmas: Experimental Evidence with Heterogenous Appropriators. Journal of Environmental Economics and Management 27:243-48.

Hardin, Garrett. 1968. "The Tragedy of the Commons." Science 162:1,243-48.

Heckathorn, Douglas D. 1993. "Collective Action and Group Heterogeneity: Voluntary Provision versus Selective Incentives," American Sociological Review 58:329-50.

Herr, Andrew. 1996. Appropriation Externalities in the Commons: Theory and Experimental Evidence, Ph.D. diss., Indiana University.

Herr, Andrew, Roy Gardner, and James Walker. 1997. "Appropriation Externalities in the Commons: Repetition, Time Dependence, and Group Size." Games and Economic Behavior, 19:77-96.

Hess, Charlotte. 1996. Common-Pool Resources and Collective Action: A Bibliography, volume 3. Bloomington: Indiana University, Workshop in Political Theory and Policy Analysis.

Kanbur, Ravi 1992. "Heterogeneity, Distribution and Cooperation in Common Property Resource Management," Policy Research working papers. Washington, D.C.: The World Bank.

Levhari, David, and Leonard J. Mirman. 1980. "The Great Fish War: An Example of Using a Dynamic Cournot-Nash Solution," Bell Journal of Economics 11:322-34.

Libecap, Gary D. 1995. "The Conditions for Successful Collective Action." In Local Commons and Global Interdependence: Heterogeneity and Cooperation in Two Domains, eds. Robert O. Keohane and Elinor Ostrom, 161-90. London: Sage Publications.

Martin, Lisa. 1995. "Heterogeneity, Linkage and Commons Problems." In Local Commons and Global Interdependence: Heterogeneity and Cooperation in Two Domains, eds. Robert O. Keohane and Elinor Ostrom, 71-92. London: Sage Publications.

McCay, Bonnie J., and James M. Acheson. 1987. The Question of the Commons: The Culture and Ecology of Communal Resources. Tucson: University of Arizona Press.

National Research Council. 1986. Proceedings of the Conference on Common Property Resource Management. Washington, D.C.: National Academy Press.

Netting, Robert McC. 1993. Smallholders, Householders: Farm Families and the Ecology of Intensive, Sustainable Agriculture. Stanford, Calif.: Stanford University Press.

Oliver, Pamela E., Gerald Marwell, and Ruy Teixeira. 1985. "A Theory of the Critical Mass. I. Interdependence, Group Heterogeneity, and the Production of Collective Action," American Journal of Sociology 91:522-56.

Ostrom, E. 1990. Governing the Commons: The Evolution of Institutions for Collective Action. New York: Cambridge University Press.

Ostrom, E., R. Gardner, and J. Walker. 1994. Rules, Games, and Common-Pool Resources. Ann Arbor: University of Michigan Press. 
Ostrom, V., D. Feeny, and H. Picht, eds. 1993. Rethinking Institutional Analysis and Development: Issues, Alternatives, and Choices. 2d ed. San Francisco: ICS Press.

Pesca (Web page of the Common Fisheries Policy of the European Union), 1998.

www. pesca.ln/en/policy8_en. html

Pinkerton, Evelyn, ed. 1989. Co-operative Management of Local Fisheries: New Directions for Improved Management and Community Development. Vancouver: University of British Columbia Press.

Reinganum, Jennifer F., and Nancy L. Stokey. 1985. "Oligopoly Extraction of a Common Property Natural Resource: The Importance of the Period of Commitment in Dynamic Games," International Economic Review 26:161-73.

Salant, Stephen W., and Eban Goldstein. 1990. "Predicting Committee Behavior in Majority Rule Voting Experiments." RAND Journal of Economics 21:293-313.

Sengupta, Nirmal. 1991. Managing Common Property: Irrigation in India and the Philippines. New Delhi: Sage.

Tang, Shui Yan. 1992. Institutions and Collective Action: Self-Governance in Irrigation. San Francisco, Calif.: ICS Press.

Thomson, James T. 1992. A Framework for Analyzing Institutional Incentives in Community Forestry. Rome, Italy: Food and Agriculture Organization of the United Nations, Forestry Department, Via delle Terme di Caracalla.

UNEP. 1991. Handbook for the Montreal Protocol on Substances that Deplete the Ozone Layer. Nairobi: Ozone Secretariat.

Wade, Robert. 1994. Village Republics: Economic Conditions for Collective Action in South India. San Francisco: ICS Press.

Walker, James A., Roy Gardner, and Elinor Ostrom. 1991. "Rent Dissipation and Balanced Deviation Disequilibrium in Common Pool Resources: Experimental Evidence," In Game Equilibrium Models II: Methods, Morals, and Markets, ed. R. Selten, 337-67. New York: Springer Verlag. 